\title{
Edge Detection with Neuro-Fuzzy Approach in Digital Synthesis Images
}

\author{
Fatma ZRIBI \\ Ecole Nationale d'Ingénieurs de Tunis (ENIT) \\ Tunis, TUNISIA
}

\author{
Noureddine ELLOUZE \\ Ecole Nationale d'Ingénieurs de Tunis (ENIT) \\ Tunis, TUNISIA
}

\begin{abstract}
This paper presents an enhanced Neuro-Fuzzy (NF) Approach of edge detection with an analysis of the characteristic of the method. The specificity of our method is an enhancement of the learning database of the diagonal edges compared to the original learning database. The original inspired NF edge detection model uses just one image learning database realized by Emin Yuksel. The tests are accomplished in synthesis images with a noised one of $20 \%$ of Gaussian noise.
\end{abstract}

Keywords-Neuro-Fuzzy; learning databases; Gaussian noise; synthesis images

\section{INTRODUCTION}

On the basis Systems Artificial Intelligence Neuro Fuzzy Neural networks are forms with the benefits of parallel processing and the learning capacity inspired from the nervous system and fuzzy logic that allows the possibility of modeling language and Cognitive systems of human decision. [1]

The systems based on neural networks and fuzzy logic are Good approximations function from sample data. They do not Need mathematical model. They are pure estimators

But they bring something more compared to the statistical Approach : they do not require a priori knowledge about the inner workings of the system.

The main feature of neural and fuzzy systems is adaptive control and statistics. They are digital estimators and dynamic systems. The neural theory drowns in the mathematical semantics of dynamic systems. Fuzzy theory overlaps with these semantic and more with probability, mathematical logic, and theories of measurement.

In general neuronal systems and fuzzy one are used to improve the performance of real systems. [2]

Neural Networks and Fuzzy Logic; both approaches belong to the large class called structures of nonlinear systems that has some properties of extreme importance on knowledge a priori of a control system. Multiple nodes interconnections are achieved and performed a learning phase by adapting the weights of nodes interconnections [2]. This process is called "adaptation" of weight. Modify the weight changes data stored in the network, in fact, the weight values represent the "memory" of the network. Neural Networks (NN) Are special models of nonlinear systems modeling of the biological nervous system. Fuzzy Logic (FL) is a system modeled by language skills and human reasoning.
In this paper, we present related works in the second part of our paper. The third part we detail the architecture of our Neuro-Fuzzy edge detection system. The next step interpreter the result obtained for noisy images to detect edges. We finish this paper with a discussion and a conclusion.

\section{RELATED WORKS}

Neuro-Fuzzy edge detection was used widely in this last decades. In [6] authors propose an ANFIS model with 8-inputs and 1-output Neuro-Fuzzy system based in first-order-Sugano system. 2 triangular type membership functions are used for each input, and the output has a constant membership function. 256 rules were used with just one output. Authors use a Grid partition method on the contrary of subtractive clustering method. The Gray level image is used to detect edges, they firstly binarize the image, and then the binary image disintegrates to $3 \times 3$ windows and generates a set of the image pattern. The edge patterns in binary images were classified into 32 categories. Training the ANFIS on this category (patterns) classify the blank elements in each $3 \times 3$ window in white pixels (value: 1) and dark pixels (value: 0 ).

In [7] authors classify image pixels into three sets of pixels contour, regular and texture using a model of Neuro-Fuzzy approach which takes the advantages of Neural Network's Learning characteristic and the fuzzy logic function. Spatial properties of the image features are the base of this approach. As every Neuro-Fuzzy system, a training set was used to create and train the classifier system. They assigned for every pixel a degree of membership for each of the three fuzzy subsets. They note that «this approach would be highly attractive in the biomedical field »

In [8] authors uses an ANFIS( Adaptative Neuro-Fuzzy Inference System) detector and « is a first-order Sugeno type Fuzzy inference system with 4-inputs and 1-output. Each input has three generalized bell type membership functions and the output has a linear membership function ». for the training they use Sugano first order model which characteristic is the combination of applying a mixature of the least-squares method and the backpropagation gradient descent method. This combination is to realize a given training data set.

Authors of the paper [9] use an ANFIS with $2 \times 2$ windows For edge detection. They allow four input values which are mapped to « the four fuzzy inputs in the fuzzy inference system ». the ANFIS system classify the output as an edge or as background. 
In [10] authors use Neuro-Fuzzy system in the classification of biometric Multimodal Face, Iris, and Finger Fake they detect this characteristic with an ANFIS system. Another application of ANFIS is used in [11] to classify Texture Image. They compare Classification with Artificial Neural Networks and ANFIS one. Their results prove that's the ANFIS system gives better classification, and the methods could be applied to medical images or defense applications.

Neuro-Fuzzy system based in Takagi-Sugeno fuzzy inference system was used in [12] as a classifier for image retrieval. They use Canny detector for the learning database.

\section{The Proposed NEURo-FuZZY EdGE DETECTION}

\section{A. Structure of the realised NF Edge Detector}

Fig.1a shows the structure of Neuro- Fuzzy detector (NF) [3]. The detector consists of four NF networks operating as sub-detectors in the four directions: vertical, horizontal, right diagonal and left diagonal direction respectively. Each detector operates on a window of the size of $3 \times 3$ pixels given in Fig. $1 \mathrm{~b}$. The NF sub-detector evaluates the relationship of the central pixel of the window with its neighborhood according to one of the four selected combinations of the contour direction. The topologies of the various selected combinations are shown in Fig.1c.

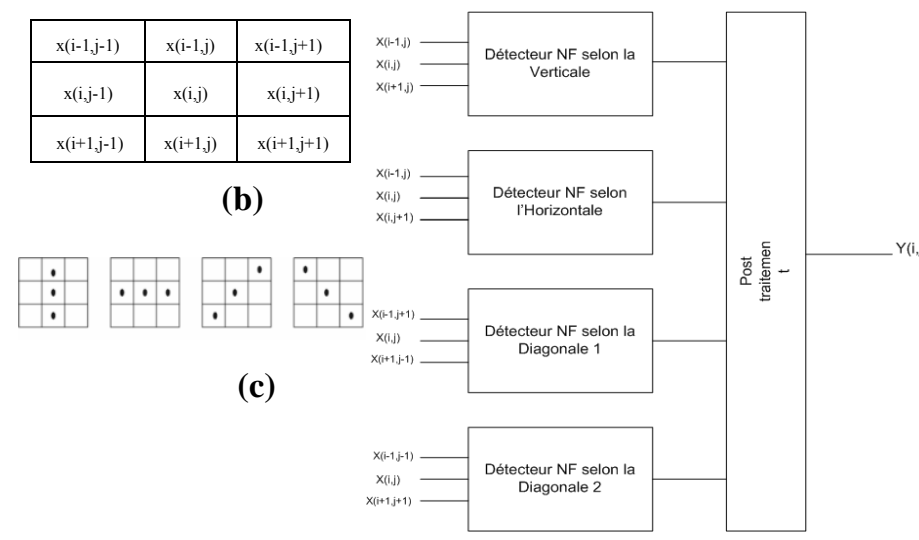

(a)

Fig. 1. (a) The structure of the neuro- fuzzy detector. (b) operator Filter window . (c) Different Topology orientations of selected contours

\section{B. Architecture of Neuro- Fuzzy detector}

A sub-NF-detector is a fuzzy inference system with 3-input and 1-Sugeno of the first order output Fig.1. The internal structures of each sub-detector are identical. Each entry has three functions of generalized Bell type of membership function. The input-output relationship of each sub-detector is governed by a base rule defined as:

$\mathrm{X} 1, \mathrm{X} 2$, and $\mathrm{X} 3$ are the inputs of the detector and $\mathrm{Y}$ is the corresponding output. The basic rule represents differently possible cases combinations between the three functions. Each function belong to each entry. It corresponds to a basic rule of 27 data laws as follows:

if (X1 is M11) and (M21 X2) and (X3 M31) then R1 = F1 $(\mathrm{X} 1, \mathrm{X} 2, \mathrm{X} 3)$ if (X1 is M11) and (M21 X2) and (X3 M32) then R2 = F2 $(\mathrm{X} 1, \mathrm{X} 2, \mathrm{X} 3)$

if (X1 is M11) and (M21 X2) and (X3 M33) then R3 = F3 $(\mathrm{X} 1, \mathrm{X} 2, \mathrm{X} 3)$

if (X1 is M11) and (M22 X2) and (X3 M31) then R4 = F4 $(\mathrm{X} 1, \mathrm{X} 2, \mathrm{X} 3)$

if (X1 is M11) and (M22 X2) and (X3 M32) then R5= F5 $(\mathrm{X} 1, \mathrm{X} 2, \mathrm{X} 3)$

if $(\mathrm{X} 1$ is $\mathrm{M} 13)$ and $(\mathrm{M} 23 \mathrm{X} 2)$ and $(\mathrm{X} 3 \mathrm{M} 33)$ then $\mathrm{R} 27=$ $\mathrm{F} 27(\mathrm{X} 1, \mathrm{X} 2, \mathrm{X} 3)$

Where: Mij is the $\mathrm{j}$-th membership function of the ith entry, given by the generalized bell-type function (equation 1 and Fig.2).

$$
M_{i j}=\frac{1}{1+\left|\frac{X_{i}-a_{i j}}{b_{i j}}\right|^{2 c_{i j}}}
$$

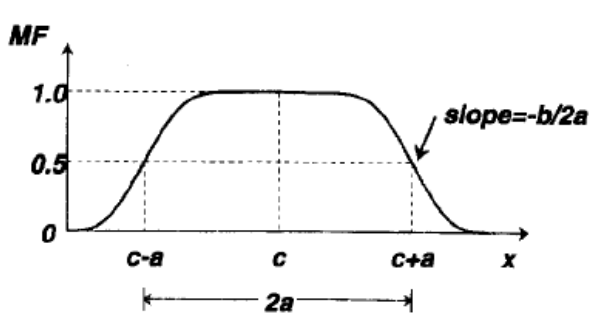

Fig. 2. Physics meaning of the parameter of The generalized Bell Function [4]

and $\mathrm{Rk}$ is the $\mathrm{kth}$ output of the rules and $\mathrm{Fk}$ is the kth output of the membership function, given by:

$$
F_{k}\left(u_{1}, u_{2}, u_{3}\right)=d_{k 0}+d_{k 1} x_{1}+d_{k 2} x_{2}+d_{k 3} x_{3}
$$

Where: $i=1,2,3 ; j=1,2,3$ and $k=1, \ldots \ldots, 27$

The parameters $a, b, c$ and $d$ are constants that characterize the shape of the different functions of the membership. The optimum values of these constants are determined by the learning phase which will be detailed in paragraph $D$.

The output of the NF detector is the weighted average for the different weights of each output of different rules (Fig3). The weighting factor $\mathrm{Wk}$. of each rule is calculated by evaluating the expression of belonging at the antecedent of the rule. This action is performed by the conversion, firstly, of the gray scale values of the input pixels in the fuzzy membership values through an initialization of the input membership Function, then the application of operator "and" to [1] Membership values. The operator "and" corresponds to a multiplication of different membership values of the entries pixels.

The weighting factors were calculated as follows: 


$$
\begin{aligned}
& w_{1}=M_{11}\left(X_{1}\right) \cdot M_{21}\left(X_{2}\right) \cdot M_{31}\left(X_{3}\right) \\
& w_{2}=M_{11}\left(X_{1}\right) \cdot M_{21}\left(X_{2}\right) \cdot M_{32}\left(X_{3}\right) \\
& w_{3}=M_{11}\left(X_{1}\right) \cdot M_{21}\left(X_{2}\right) \cdot M_{33}\left(X_{3}\right) \\
& w_{27}=M_{13}\left(X_{1}\right) \cdot M_{23}\left(X_{2}\right) \cdot M_{33}\left(X_{3}\right)
\end{aligned}
$$

Once the weighting factors are calculated, the output of the sub NF detectors are calculated using the formula (4)

$$
Y=\frac{\sum_{k=1}^{27} w_{k} R_{k}}{\sum_{k=1}^{27} w_{k}}
$$

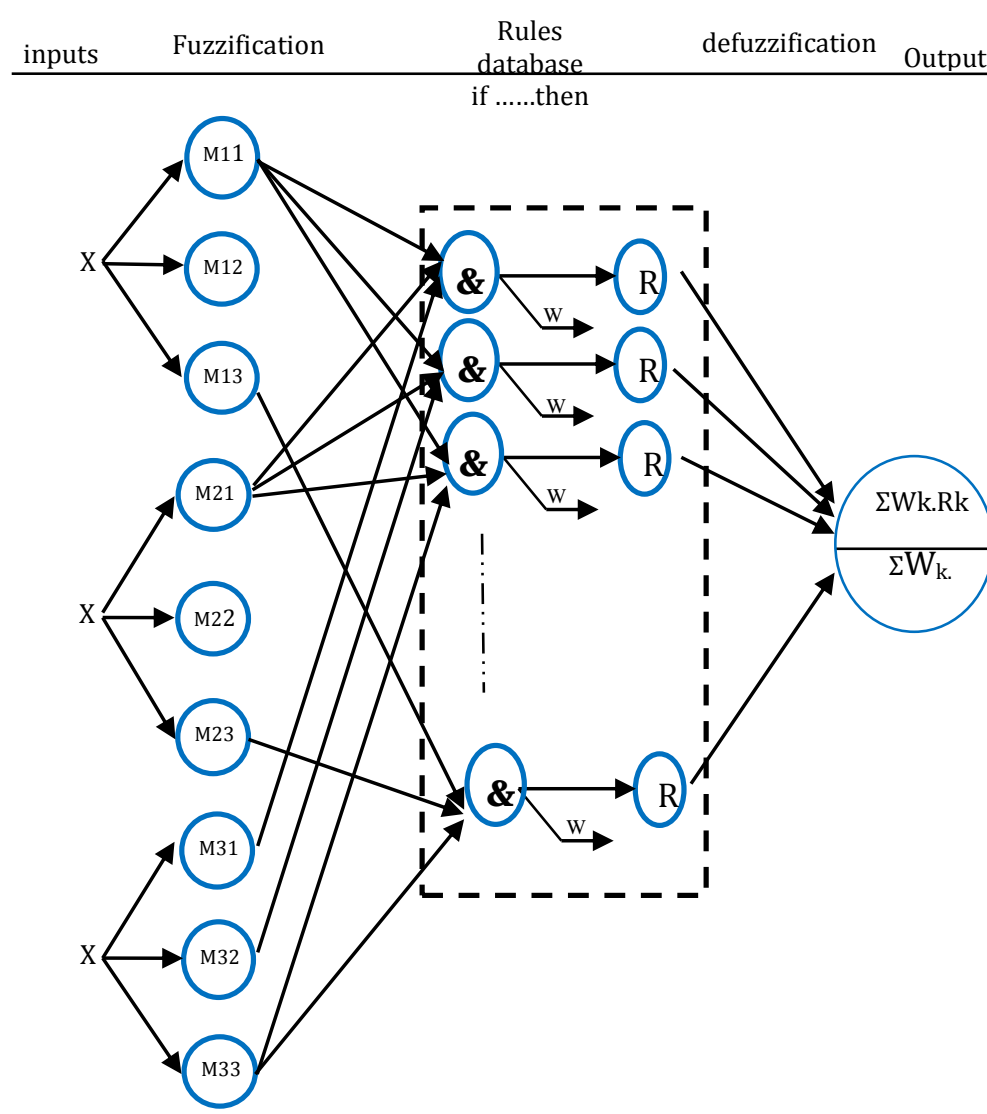

Fig. 3. The first order Sugeno model of 3 inputs and 1- output [5]

\section{Post-processing: choice of edge pixel}

This step involves a thresholding of the average of the Outputs of the various sub-detectors. An initial value of the Threshold 128 was used [3]. Following empirical tests the Threshold value selected from the results realized, and is 90 . The input-output of the post-treatment phase relationship can be presented as follows:

Let the outputs respectively representing the four subdetectors Forming the NF network. The final decision for a given pixel corresponds to the output of the post-processing Block (Figure 1a). This output was calculated in two steps. The first is the calculation of the average of the output of the various sub-detectors was given by equation 5 ,

$$
Y_{\text {Moy }}=\frac{1}{K} \sum_{k=1}^{K} Y_{k}
$$

the second step is thresholding performing the decision to set the belonging to the appropriate edge (Equation 6).

$$
y(r, c)= \begin{cases}0 & \text { if } Y_{\text {Moy }}<90, \\ 255 & \text { else }\end{cases}
$$

$y(r, c)$ is the output of the post-processing block corresponding to the output of the NF detection operator.

\section{The Learning of the various sub-detectors}

The internal parameters of the NF detector were optimized by a learning phase which is carried out independently for each sub-detector of the overall structure (Figure 1a). The Figure 4 presents the diagrams of the learning process for a given NFsub-detector.

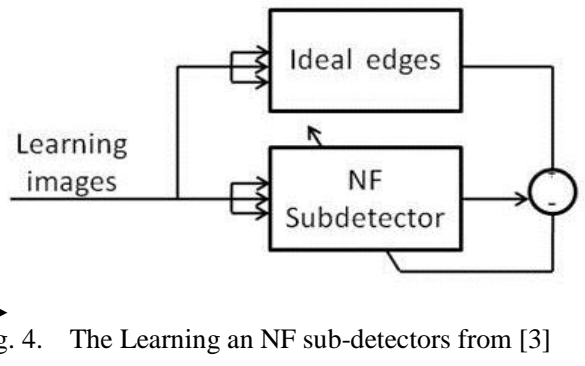

The selected learning database consists of two images: one for learning vertical and diagonal contours and the second for learning the edges located on the left and right diagonal. Here in the following statistical analysis of different existing patterns in the image of learning and degree of the occurrence of different patterns. Motifs circled in red in Figure 6 represent the most contours models based on the learning, their degree of occurrence percentages are given in Figure 7.

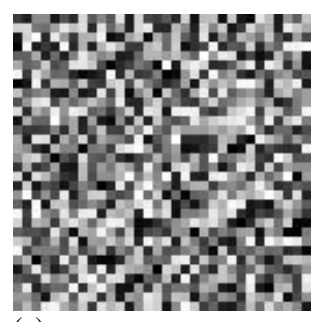

(a)

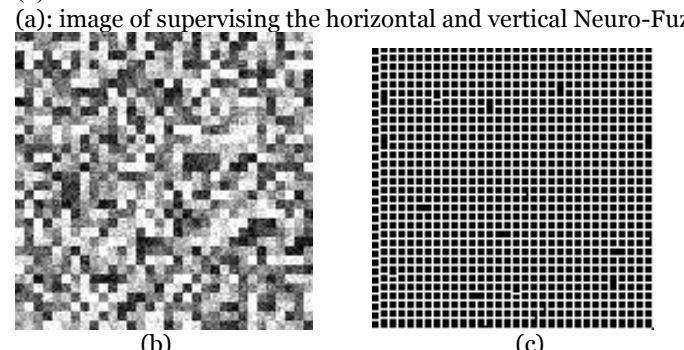

(b): image (a) blurred with Gaussian noise with $20 \%$ densities

(c): image of ideal edge (a) detected with the wavelet multi-scale product 


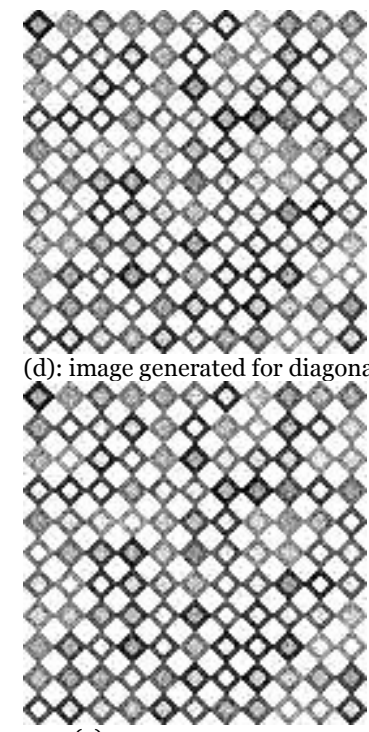

(e)

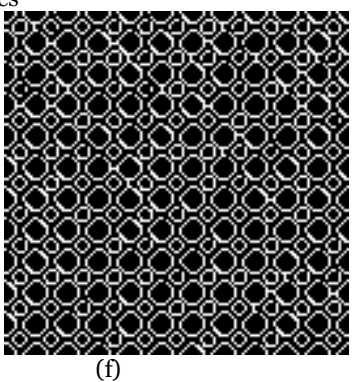

(f) (e): Image (d) blurred with Gaussian noise with density of 0.02

(f): ideal edges detected with Sobel filter for the supervision of the diagonal subdetector

Fig. 5. images of learning databases: (a) and (d) Automatic images generated with grayscale randomly between 0 and 255. (b) and (e) the correspondent images of (a) and (d) corrupted with Gaussian noise of 0.02 intensity. (c) and (f) the edges correspondent to (a) and (d) used to supervise the Neuro-Fuzzy subdetectors: (b) and (c) for the vertical and horizontal subdetectors. (e) and (f) for the diagonal subdetectors

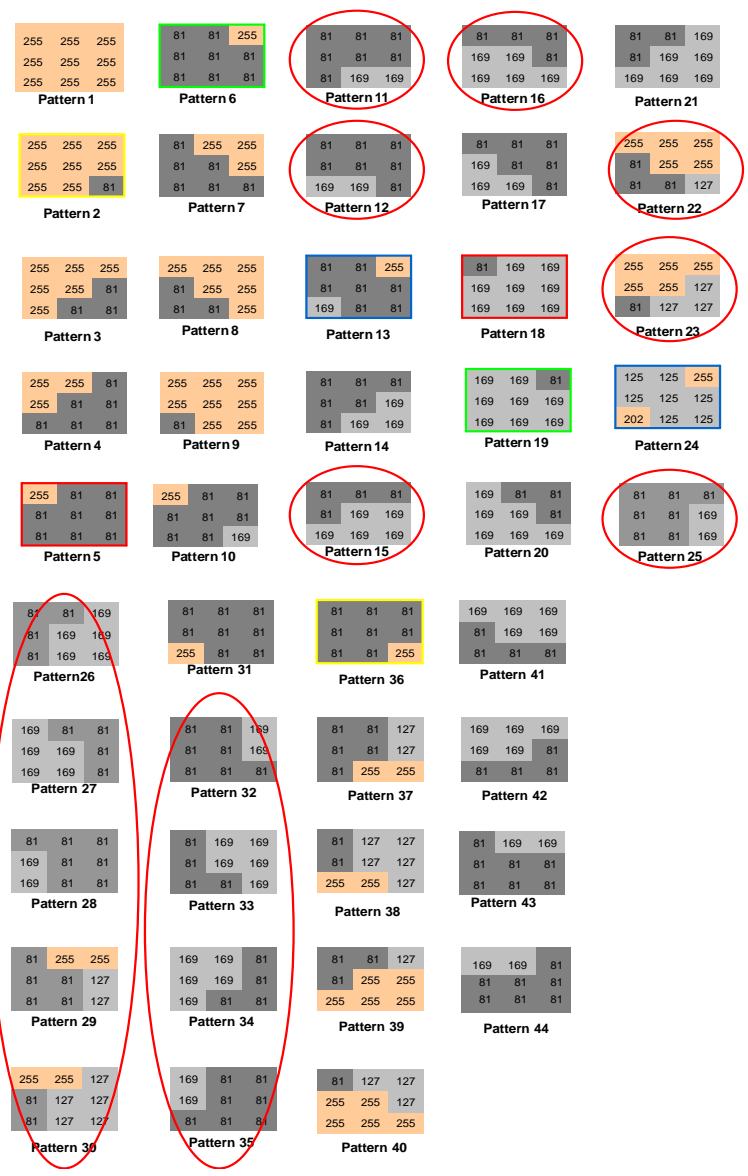

Fig. 6. Tthe most contours model based on the learning

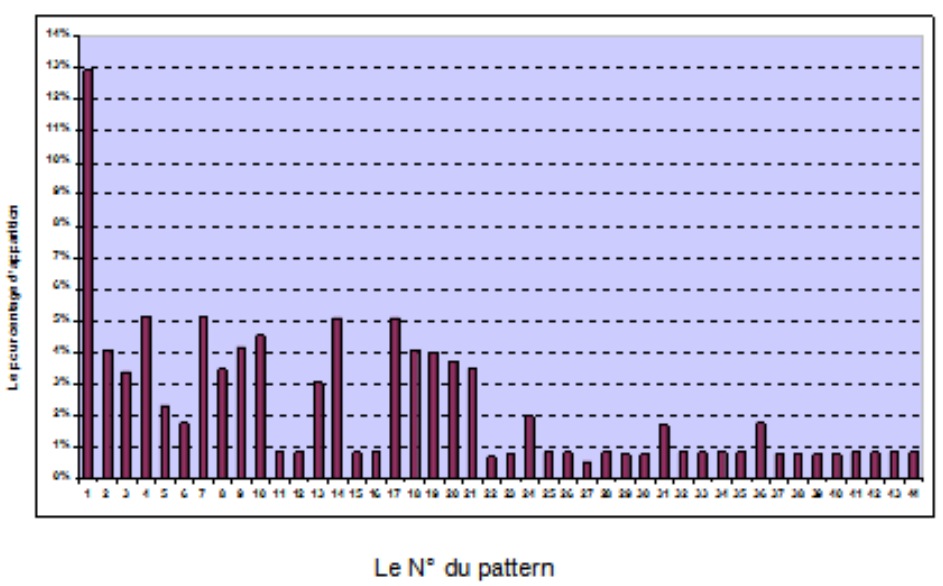

Fig. 7. The degree of occurrence percentages

Figure 6 and 7 present the degree of apparition of each pattern of our enhanced database. The pattern 1 represent the most pattern one which appears in the two databases horizontal and vertical one in one way and the two diagonal pattern of our the contribution of the database, the appear percentage is .13\%. Patterns 4, 7,14 and 17 represent a rate of apparition of 5\% Patterns number 14 and 17 has the ratio of apparition of 5\% from the hole database vertical, horizontal and two diagonal. The patterns which have a rate of $1 \%$ are: $11,12,15,16,22,23,25,26,27,28,29,30,32,33,34$ and 35 .

\section{RESULTS AND INTERPRETATION IN PRESENCE OF NOISE}

The Neuro-Fuzzy detector was tested on synthetic images such as a circle, Lena, blobs, house and a cameramen (figure 8). All images are grayscale images coded on 8 - bit size $256 \times 256$ pixels. Tests were performed on the images without noise and the same figures noised by Gaussian noise of $14 \mathrm{~dB}$ equivalent to $20 \%$ of the figure. The results of detections were compared with the detection of the same images by Sobel operator. The main feature of these detectors is revealed by the results. Indeed, the detection results on noisy images are clearly better than those given by the conventional Sobel detector.

\section{DISCUSSION}

The Neuro-Fuzzy edge detector in this paper is an example of edge detector belonging to a new family, which has the following advantages:

1) The preprocessing phase which was the subject of Several studies are no longer necessary with this detector. Just know the type of noise in the image and to learn the detector and thus, design the appropriate one.

2) Its structure is flexible. Indeed the number of sub sensor is variable depending on the accuracy in the direction of the Desired contour. Certainly, the number of sub-detector is important to the edges accuracy is fine, for cons, the running time and the computational complexity of the Algorithm is more important than the classical detector. Hence, a performance/precision compromise is needed and time depends on the empirical Results performed. Such is the 
case of detection of ongoing work by the Neuro-Fuzzy approach with different numbers of detectors under (4.8 under detectors) on biomedical images: scanners and X-ray.

3) The structure of the sensor remains simple, since it consists of a Neuro-Fuzzy fundamental block of 3-inputs and 1-output.

4) The two bases are chosen of learning: is an image of the bibliography realized by Emin Yuksel and the second one proposed is our contribution for diagonal edges and it represent our contribution. These two images forming the learning base is easily generated on a machine and constitute The entire contour models possible on a $3 \times 3$ window. Analyzes and tests studying the different bases of learning will be the resultant of a paper being prepared.

\section{CONCLUSION}

We can conclude that this detector showed effectiveness are mainly for the treatment of noisy images while performing the actual detection. Many features of this sensor made so that it performs the filtering operation in parallel with the edge detection by the features of the Neuro-Fuzzy system. This characteristic was based on the property of the learning database which recognizes contours in spite of the presence of noise. Indeed, learning step is the main feature that makes learning The system settings that will recognize contours distorted by noise. These results improve that hybrid approach is better in an edge detection that conventional one especially in noisy images and it is possible to apply them in more complex images like medical ones or defense images.

\section{REFERENCES}

[1] F. L. Lewis, J. Campos and R. Selmic, "Neuro-Fuzzy Control of Industrial Systems with Actuator Nonlinearities (Frontiers in Applied Mathematics)", Society for Industrial and Applied Mathematics, April 2002 .
[2] Y. Morere, "Les Réseaux Neuro-Flous", Thèse de doctorat, L.A.M.I.H, 17 may 2001.

[3] M.E Yüksel and E.Besdok, "A simple neuro-fuzzy impulse detector for efficient blur reduction of impulse noise removal operators for digital images", IEEE Transactions on fuzzy systems, Vol. 6,N.12, pp. 854-865, 2004.

[4] J. S Roger Jang and C. T. Sun, "Neuro-Fuzzy Modeling and Control", Proceedings of the IEEE, Vol. 83, $\mathrm{N}^{\circ}$. 3, March 1995.

[5] J.-S.R. Jang , C.-T Sun and E. Mizutani , "Neuro-fuzzy and soft computing.. a computational approach to learning and machine intelligence", Prentice-Hall, Upper Saddle River, NJ, (614 pages) 1997

[6] Suryakant and Renu Dhir , " Novel Adaptive Neuro-Fuzzy based Edge Detection Technique ", International Journal of Computer Applications (0975 - 8887) Volume 49- No.4, July 2012.

[7] R. J. Oweis and M. J. Sunna, " A COMBINED NEURO-FUZZY APPROACH FOR CLASSIFYING IMAGE PIXELS IN MEDICAL APPLICATIONS, Journal of ELECTRICAL ENGINEERING, VOL. 56, NO. 5-6, 2005, 146-150

[8] Lei Zhanga Mei Xiaoa Jian Maa Hongxun Song, "A Novel Edge Detection Method Based on Adaptive Neuro-Fuzzy Inference System ", PIAGENG 2009: Intelligent Information, Control, and Communication Technology for Agricultural Engineering, edited by Honghua Tan, Qi Luo, Proc. of SPIE Vol. 7490, 74902S @ 2009 SPIE - CCC code: 0277786X/09/\$18 - doi: 10.1117/12.836828, Proc. of SPIE Vol. 7490 74902S-1

[9] S. Anwar and S. Raj, "A Neural Network Approach to Edge Detection using Adaptive Neuro-Fuzzy Inference System", International Conference on Advances in Computing,Communicationsand Informatics (ICACCI), 2014.

[10] S. Wilson and A. Lenin Fred, "An Efficient Biometric Multimodal Face, Iris and Finger Fake Detection using an Adaptive Neuro Fuzzy Inference System (ANFIS)", Middle-East Journal of Scientific Research 22 (6): 937-947, 2014

[11] S. Panigrahi, T. Verma, "Texture Image Classification using Neurofuzzy Approach", International Journal Of Engineering And Computer Science ISSN:2319-7242 Volume 2 Issue 7, Page No. 2309-2313, July 2013.

[12] S.Asha, S.Ramya, M.Sarulatha, M.Prakasham, P.Priyanka," Neuro Fuzzy Classifier for Image Retrieval", International Journal of Innovative Research in Computer and Communication Engineering, Vol. 3, Issue 1, January 2015. 

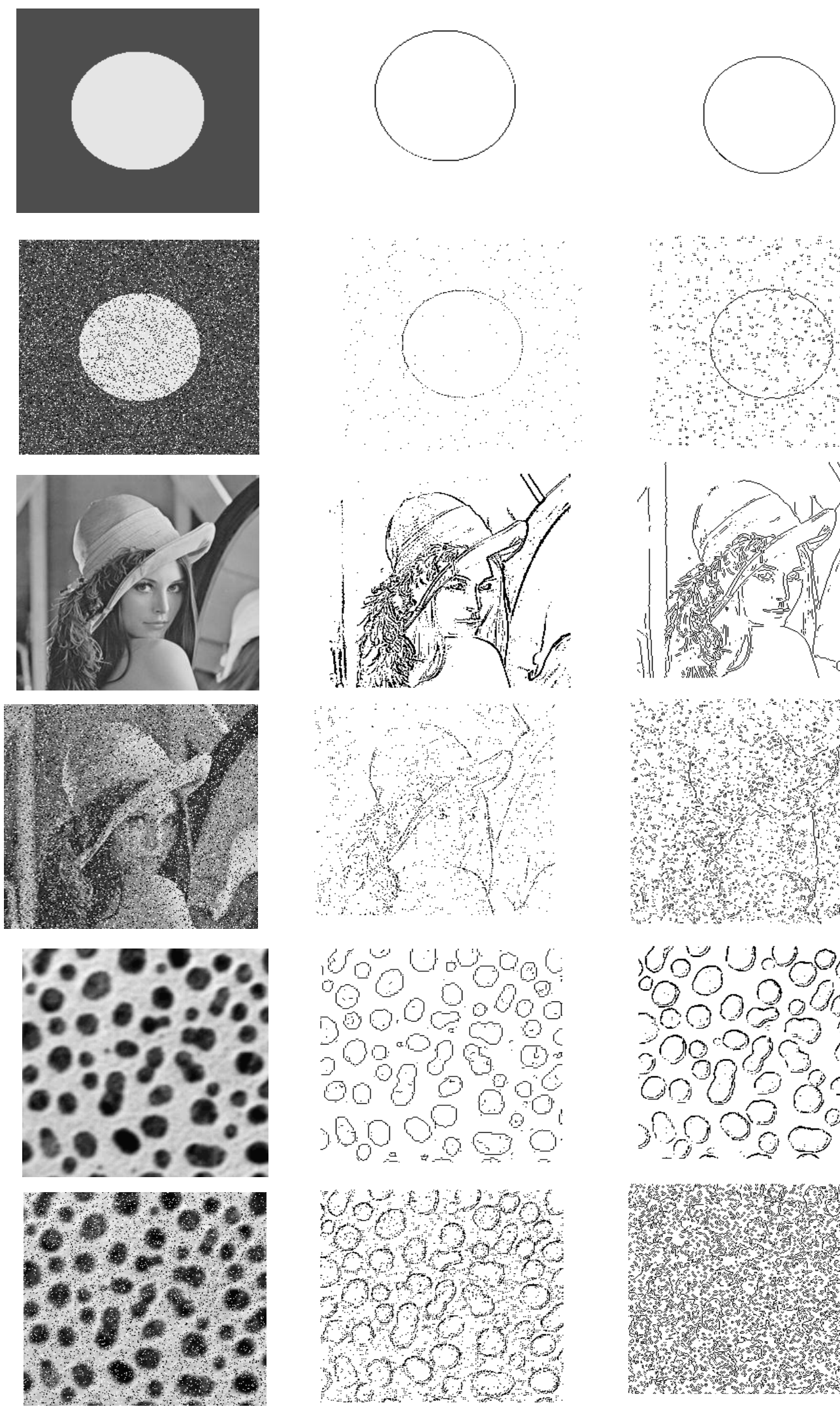
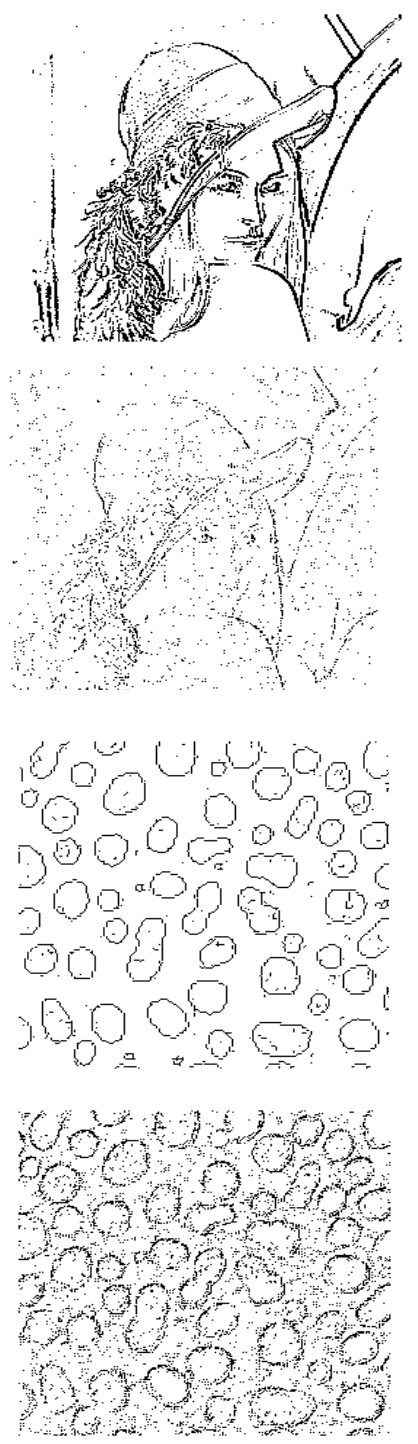
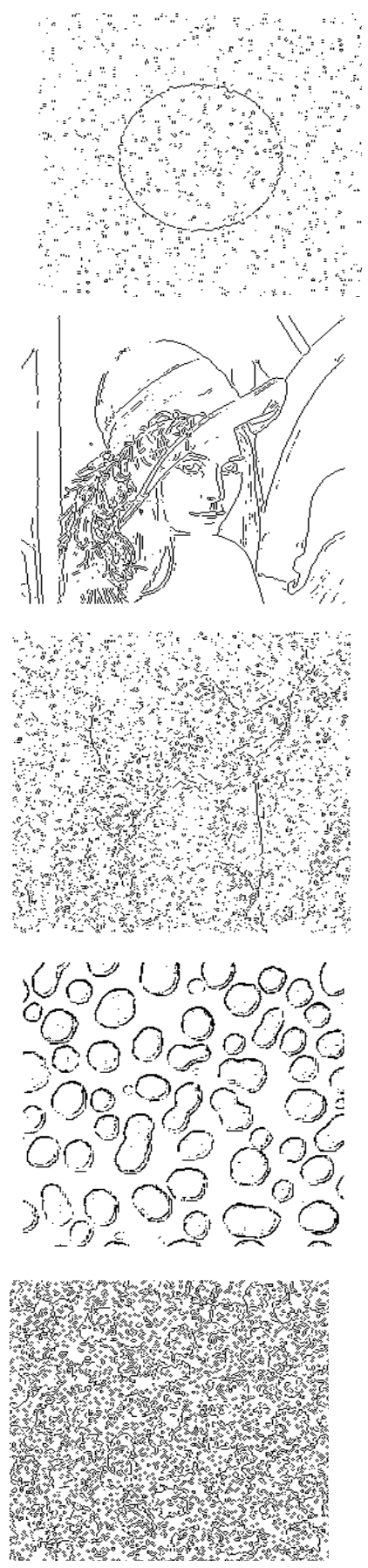

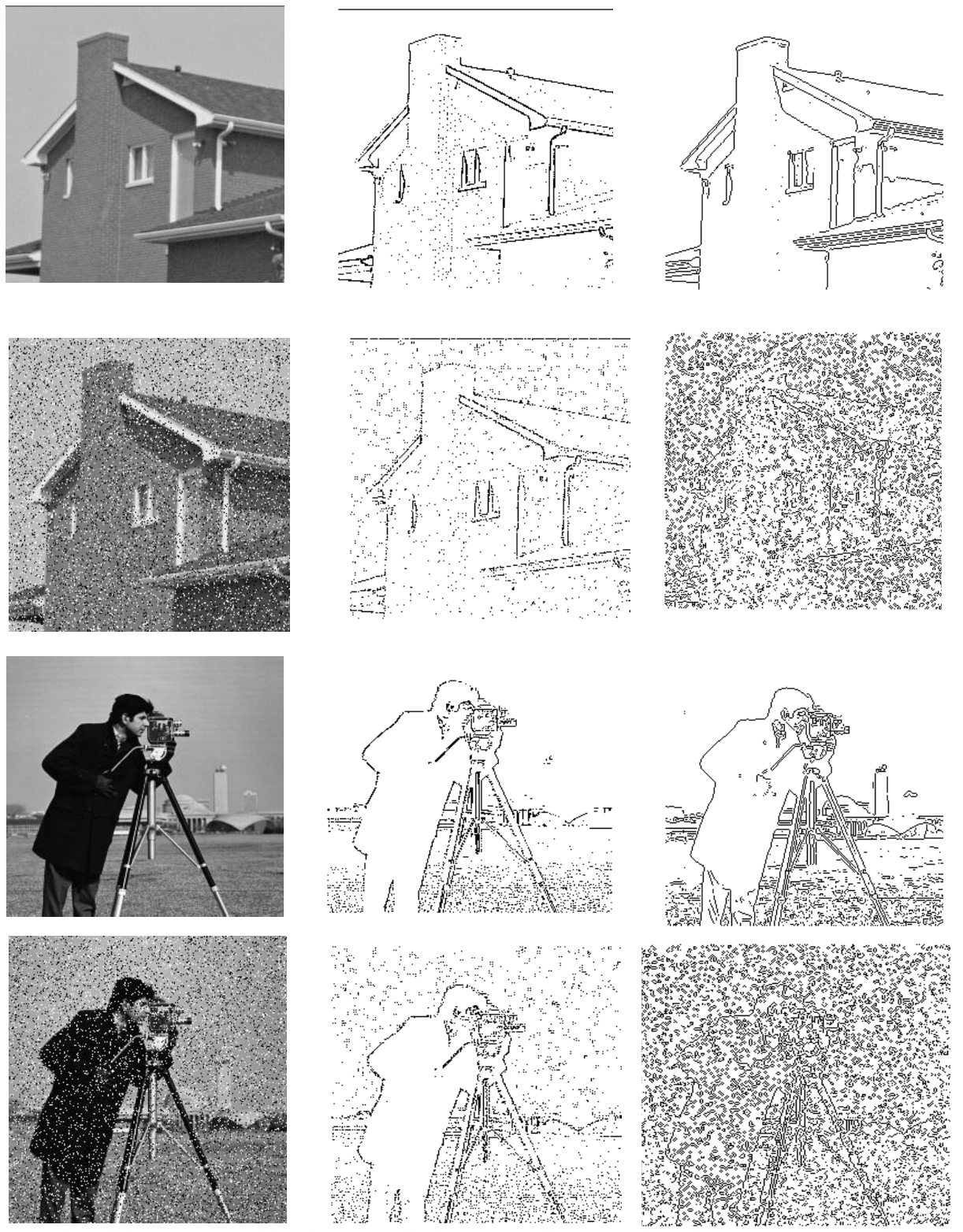

Fig. 8. Left : Original image tracking the same noisy image, medium: detection with the Neuro- Fuzzy detector, Right : Detection with Sobel detector 